\title{
Horse trials and tribulations: Balancing breeding, performance and welfare metrics
}

J Murphy

University College Dublin, Dublin, Ireland

Email:jack.murphy@ucd.ie

Introduction Man has domesticated many animal (and plant) species primarily for the benefit of human culture in one form or another. As distinct from animals bred predominantly for the provision of food or those designed purely to function as companions or pets, the horse is generally bred to participate in one or more competitive sporting activities (Murphy \& Wall, 2009). While others fulfil the role of companions, pets and/or workmates and even partners in some instances, the horse is commonly referred to as an athlete. Indeed, equine athletes are often the primary attraction in popular spectator sports such as horse racing, showjumping, polo and dressage among others, where humans participate as riders, drivers and trainers (Murphy \& Arkins, 2007). The equestrian sports regularly attract very significant financial investment, ownership status, sponsorship arrangements and the opportunity to win or earn what is often very lucrative prize money, kudos and iconic status for man and beast.

There is however, a sector of society that espouses the view that these purpose bred sporting creatures are little more than entertainers or animal gladiators. This particular societal view contends that the animals' participation in the chosen sport is at best ambiguous. Their involvement on a truly voluntary basis may be suspect where there is easy justification for the ready disposal of animals when they have passed their 'sell by date'. Some thirty years ago, Jeffcott et al. (1982) published the initial assessment of British Thoroughbred breeding and racing, which examined the reasons why racehorses failed to fulfill their potential to train and race. Indeed, the term 'wastage' has been used to describe the phenomenon of unwanted surplus due to failure to compete in several subsequent equine studies (Rossdale et al. 1985; Bailey et al. 1997; Olivier et al. 1997). These 'wastage' studies, which detailed the causes and extent of horses failing to compete, have been useful in terms of evaluating athletic performances and reproductive success of the horses. But little work has been undertaken to address the increasingly important issues of over-breeding, under-performance and welfare concerns within the wider horse industry. Indeed, there now appears to be a growing issue of public concern aimed at the wider industry in terms of what happens to horses when they fail to train and/or perform (Murphy \& Wall 2009). In reality, there are several different circumstances and conditions that could lead to athletic performances deemed as lack lustre or insufficient, or when what were previously successful careers decline due to the occurrence of injury/disease or some other issue that either radically reduces or terminates the animal's value or utility worth for owner/breeders.

In keeping with the Roman gladiator analogy, the success of some animal field sports (for example game birds) depends upon killing the animal in the pursuit of sport. Although not the case in any horse related activity, death happens on occasion and such fatal accidents are often reported as high profile incidents in the media. In fact, these events and the dearth of reliable data (including valid scientific studies on risk factors affecting retirement) on the fate of horses when they leave their sporting careers alive is often highlighted by societal indifference to or condemnation of many horse sports. For example, Animal Aid actively campaigns against horse racing through their official website (www.animalaid.org.uk) and provides statistics of fatal horse injuries that occur across UK racecourses. As in any formal breeding programme, breeding equine athletes or other animal gladiators is a game of skill and chance involving basic animal science principles. Generally, the primary goal is to breed horses that will win races or succeed in competition. However, the question must be asked if the human owner rather than the horse per se is the real winner? There may well be a fundamental assumption on the part of the breeder at the outset that his/her horses will always be well cared for and be useful and adaptable athletes. Every horse breeder dreams of breeding the horse with great potential for high level performance as an athlete in some equine career. However, as is the case with all normal distributions, all horses simply cannot be elite and most horses are merely average in terms of ability with only very few truly talented horses in any of the equestrian disciplines. This issue now poses a serious threat and presents an immediate challenge to the horse racing authorities and other equine governing bodies/federations. These institutions must all share in the application of proper scientific techniques to benefit the horse by reducing over-production and unnecessary wastage and improving welfare provisions.

Conclusion Humans have capitalized on the evolution of the horse to fulfil many of mans' sporting pleasures. The horses' innate motivation to gallop and ready acceptance of training has provided kudos, enjoyment and financial reward for countless breeders, owners and handlers. While the elite equine athletes are much admired individuals - often achieving legendary status, current societal standards demand immediate action to balance some indiscriminate breeding objectives, performance demands and appropriate welfare for the good of the horse.

\section{References}

Bailey, C.J., Rose, R.J., Reid, S.W.J. and Hodgson, D.R. 1997. Wastage in the Australian Thoroughbred racing industry: a survey of Sydney trainers. Australian Veterinary Journal 75, 64-66.

Jeffcott, L.B., Rossdale, P.D., Freestone, J., Frank, C.J. and Towers-Clark, P.F. 1982. An assessment of wastage in Thoroughbred racing from conception to 4 years of age. Equine Veterinary Journal 14, 185-198.

Murphy, J. and Arkins, S. 2007. Equine Learning Behaviour. Behavioural Processes.

doi.org/10.1016/j.beproc.2006.06.009

Murphy, J. and Wall, P. 2009. Overproduction of Irish horses: Implications for equine health, equine welfare and public health. In: Agricultural Research Forum, pp. 79. Tullamore: Standard Printers, Galway.

Olivier, A., Nurton, J.P. and Guthrie, A.J. 1997. An epizootological study of wastage in Thoroughbred racehorses in Gauteng, South Africa. Journal of the South African Veterinary Association 68, 125-129.

Rossdale, P.D., Hopes, R., Wingfield-Digby, N.J. and Offord, K. 1985. Epidemiological study of wastage among racehorses 1982 and 1983. Veterinary Record 116, 66-69. 